\title{
Partially Corrective AdaBoost
}

\author{
Yan Jiang and Xiaoqing Ding \\ Department of Electronic Engineering, Tsinghua University, Beijing, China, 100084
}

$\{$ jyan, dxq\}@ocrserv. ee.tsinghua.edu.cn

\begin{abstract}
We propose a novel algorithm to improve the ensemble performance of AdaBoost. The main contribution in our algorithm includes two aspects: (1) we aim to generate a distribution at each step that has less correlation with the previous classifiers so as to enhance the searching efficiency for new classifiers; (2) the classifiers weights can be iteratively modified along with the training process. In the proposed algorithm, the distribution is required to be corrective to some previous classifiers or some previous classifiers' linear combinations. Experiments on UCI Repository have validated the new algorithm's effectiveness.
\end{abstract}

\section{Introduction}

AdaBoost is a meta-algorithm, proposed by Y. Freund and R. E. Schapire ([1]) as an example to aggregate weak classifiers into a strong one in probably approximately correct (PAC) learning framework. A single weak classifier is only required to perform slightly better than random guess to assure the combined classifier is able to achieve arbitrarily good bounds on its training error.

Theoretical analysis reveals that the AdaBoost can be regarded as a gradient descent process in functional space ([2]) that minimizes a cost function, which is the upper bound of the training error. Based on the analysis, some researchers have suggested different ways to construct a more effective descent direction in order to get a faster convergence performance. For example, Friedman et al. ([3] use Newton step in LogitBoost and GentleBoost and Li Ling pursues a conjugate gradient direction in his CGBoost to accelerate the training ([4]).

On the other hand, AdaBoost can also be regarded as an entropy projection process, which means the new distribution should be very 'close' to the the last in the sense of Kullback-Leibler divergence subjected to the constraint that requires the new distribution to be 'corrective' to the current classifier ([5]).

There are two main problems lies in AdaBoost: (1) the constraint for the next distribution is only designed to reduce the correlation between two adjacent member classifiers; (2) the member classifiers' coefficients can not be modified once assigned. The first problem results in the fact that the newly added member classifier, although uncorrelated to the last, is very similar to some previous classifiers. It may potentially counteract the searching efficiency for new member classifiers. Intuitively, the second problem will always weaken the ensemble performance since some previously assigned coefficients are no longer suitable when a new member is added. This can also be understood from a statistical view. In 
[6], AdaBoost is extended to regression model and regarded as a typical forward stage-wise variable selection method. However, this variable selection strategy is proved to be inferior to forward step-wise variable selection method, which iteratively modifies all the model coefficients once a new variable is selected.

\section{Preliminaries}

We are give $N$ labeled training samples $\left\{\left(\mathbf{x}_{i}, y_{i}\right)\right\}_{1 \leq i \leq N} \subset \mathcal{X} \times\{-1,+1\}$. AdaBoost selects one classifier $h_{t}: \mathcal{X} \rightarrow\{-1,+1\}$ at $t$-th round and assign a coefficient $\alpha_{t}$ to it. After $T$ rounds of training, AdaBoost finally outputs a classifier ensemble $H_{T}=\operatorname{sgn}\left(F_{T}(\mathbf{x})\right)$, where $F_{T}(x)=\sum_{t=1}^{t=T} \alpha_{t} h_{t}(\mathbf{x})$.

\subsection{Boosting Is a Gradient Descent Process}

AdaBoost is analyzed as a gradient descent process to minimize a cost $C\left(F_{t}\right)([2])$.

$$
C\left(F_{t}\right)=\log \sum_{i=1}^{i=N} \exp \left(-y_{i} F_{t}\left(\mathbf{x}_{i}\right)\right)
$$

The gradient of $C\left(F_{t}\right)$ is denoted by $\nabla C\left(F_{t}\right)$ and its $p$-th component can be calculated as Eq.(2), where $d_{t}(p)$ is the $p$-th sample weight at $t$-th iteration.

$$
\left.\nabla C\left(F_{t}\right)_{p} \triangleq \frac{d C\left(F_{t}+s 1_{\mathbf{x}_{p}}\right)}{d s}\right|_{s=0}=-y_{p} d_{t+1}(p)
$$

A new member classifier is required to be as 'close' as possible to the negative gradient direction of $C\left(F_{t}\right)$ in the sense of inner product (Eq. (3) ). This process is equivalent to minimize the error rate according to the new distribution.

$$
\left\langle-\nabla C\left(F_{t}\right), h_{t+1}\right\rangle=\sum_{i=1}^{i=N} h_{t+1}\left(\mathbf{x}_{i}\right) y_{i} d_{t+1}(i)=1-2 \varepsilon_{t+1}
$$

$h_{t+1}$ is selected as the new descent direction and we use exact line search to determine the step size (Eq.(4)), which is also the coefficient assigned to $h_{t+1}$.

$$
\alpha_{t+1}=\underset{\alpha \in \mathbb{R}}{\arg \min } C\left(F_{t}+\alpha h_{t+1}\right)
$$

\subsection{Boosting as Entropy Projection and Totally Corrective AdaBoost}

In [5], at each iteration, the new distribution is regarded as the 'projection' onto the hyperplane $\Delta_{t}$ (Eq.(5)). This actually gives the Lagrange dual of Eq.(4). The 'corrective' constraint of the hyperplane requires the new distribution makes the current weak hypothesis uncorrelated with the training samples. Therefore, the new classifier $h_{t+1}$ must have some new classification behaviors compared with 
$h_{t}$. It guarantees that AdaBoost can generate a sequence of diverse classifiers that is essential to the performance of the ensemble. However, as we have discussed, one 'corrective' constraint is not enough to adequately reduce the correlations between the new member classifier and all the previous ones.

$$
\begin{gathered}
\Delta_{t} \triangleq\left\{\mathbf{u} \in \mathbb{R}^{N} \mid \sum_{i=1}^{i=N} y_{i} h_{t}\left(\mathbf{x}_{i}\right) u_{i}=0, \sum_{i=1}^{i=N} u_{i}=1, u_{i} \geq 0, \forall 1 \leq i \leq N\right\} \\
\mathbf{d}_{t+1}=\underset{\mathbf{u} \in \Delta_{t}}{\arg \min } \sum_{i=1}^{i=N} u_{i} \log \frac{u_{i}}{d_{t}(i)}
\end{gathered}
$$

Intuitively, we can obtain a more effective classifier that has less correlation with the already available classifiers by simply extending the 'corrective' requirement to all the previous classifiers (Eq.(7D). This is exactly the basic idea of totally corrective AdaBoost (TC-AB) ([5]). The dual problem of Eq.(6) subjected to the 'totally corrective' constraint Eq.(7) is Eq.(8). Consequently, we must modify the classifiers' weight as $F_{t}=F_{t-1}+\sum_{\tau=1}^{\tau=t} \alpha_{t, \tau} h_{\tau}$. TC-AB actually uses all the previous classifiers to construct the descent direction to minimize the same cost function as AdaBoost, where a 'good' descent direction is adopted.

$$
\begin{gathered}
\sum_{i=1}^{i=N} u_{i} y_{i} h_{\tau}\left(x_{i}\right)=0, \quad \forall 1 \leq \tau \leq t \\
\boldsymbol{\alpha}_{t}=\underset{\boldsymbol{\alpha}=\left(\alpha_{1}, \alpha_{2}, \ldots, \alpha_{t}\right) \in \mathbb{R}^{t}}{\arg \min } C\left(F_{t-1}+\sum_{\tau=1}^{\tau=t} \alpha_{\tau} h_{\tau}\right)
\end{gathered}
$$

There are two main problems lie in TC-AB. Firstly, it is always not practical that the 'totally corrective' constraints have feasible solution; secondly, the number of the 'corrective' constraints continuously increases when more member classifiers are aggregated. So TC-AB is computationally expensive and its complexity can not be controlled. Inspired by the above discussion, we mainly focus on how to improve the cost function minimization by incorporating more information from previous member classifiers. Besides, the computational complexity of the developed algorithm can be adequately controlled.

\section{Partially Corrective AdaBoost}

In this section, we build a 'partially corrective' distribution instead by relaxing the 'totally corrective' constraints. We provides two different implementations for partially corrective AdaBoost (PC-AB).

\subsection{Two Types of Partially Corrective AdaBoost}

In the first case (PC-AB1), the new distribution is only required to be corrective to some of the previous classifiers(Eq.(9)). In the second case (PC-AB2), we 
construct a set of linear combinations of all the previous classifiers in advance and force the new distribution to be 'corrective' to these combinations(Eq. (10) ).

The dual problems of PC-AB1 and PC-AB2 can be summarized in Eq.(11a) and Eq. 12b respectively. PC-AB1 selects a subset from the previous classifiers to construct a new descent direction and the combination coefficients are determined by exact line search. PC-AB2 constructs a set of candidate directions by linearly combining all the classifiers (Eq. (12a) ) and also uses exact line search to decide how to combine these directions (Eq.(12b)). It's easy to check that the distribution calculated from Eq.(9) satisfies Eq.(11b) and the distribution calculated from Eq.(10) also satisfies Eq.(12c). Consequently, member classifiers' coefficients must be backward adjusted respectively (Eq.(11c) and Eq.(12d) $)$. Thus we can see that the requirement for a new distribution that is less correlated to the previous member classifiers is intrinsically consistent with the adjustment of member classifiers' coefficients from Lagrange duality.

$\mathrm{PC}-\mathrm{AB}$ is regarded as a tradeoff between the requirement of time complexity and the adequate consideration of the previous classifiers' information. It is easy to see that AdaBoost and TC-AB can be summarized as special cases of PC-AB1; further, PC-AB2 implements a more general idea of PC-AB1. Actually, CGBoost is categorized as a special form of PC-AB2's idea, but it utilizes only one linear combination as the descent direction. Since our attempts in PC-AB1 and PCAB2 aim to adopt a more effective descent direction in minimizing the same cost function as AdaBoost(Eq.(1)), it is easy to understand that we have the same training error bound as AdaBoost. But we can always achieve faster decreasing training errors because we optimize the cost function more aggressively.

$$
\begin{aligned}
& \sum_{i=1}^{i=N} d_{t+1}\left(\mathbf{x}_{i}\right) y_{i} h_{t_{k}}\left(\mathbf{x}_{i}\right)=0,1 \leq t_{k} \leq t, 1 \leq k \leq m \\
& \sum_{i=1}^{i=N} d_{t+1}\left(\mathbf{x}_{i}\right) y_{i}\left(\sum_{\tau=1}^{\tau=t} \omega_{t, k, \tau} h_{\tau}\left(\mathbf{x}_{i}\right)\right)=0,1 \leq k \leq m
\end{aligned}
$$

\subsection{Diversity and Heuristic Classifier Subset Selection for PC-AB1}

The problem in PC-AB1 is how to choose a representative classifier subset in order to achieve a good descending performance. An intuitive idea is that the selected classifiers should have the following properties: (1) the selected classifiers should have good classification performance considering the current distribution;

(2) the correlations among the selected classifiers should be small. These two points can be explained from a computational view: the first requires that all the selected directions are 'close' to the negative gradient and the second requires that these directions are also nearly orthogonal. Due to great time requirement, it is not practical to try all the classifier subsets to find the best choice. So we propose a heuristic classifier selection strategy for PC-AB1(Fig,1).

The discrepancy measure to evaluate two classifiers' similarity here is 'diversity'. Diversity and classification rate are regarded as two important factors in classifier ensemble. The latter is always measured by the error rate while 
Input: $t$ available classifiers $h_{1}, h_{2}, \ldots, h_{t}$ and a positive integer $m$, where $1 \leq m \leq t$. Initialization: Initialize the candidate set as $C=\left\{h_{t}\right\}$.

Repeat: Choose a classifier $h_{\tilde{k}}$ from $\left\{h_{1}, h_{2}, \ldots, h_{t}\right\} \backslash C$ that satisfies Eq. (13) and add it to $C \cdot \gamma>0$ is a constant, $\operatorname{diver}(\bullet, \bullet)$ is a diversity function and $\varepsilon_{t}(h)=\sum_{h\left(\mathbf{x}_{i}\right) \neq y_{i}} d_{t}(i)$.

$$
h_{\tilde{k}}=\underset{h \in\left\{h_{1}, h_{2}, \ldots, h_{t}\right\} \backslash C}{\arg \max } \frac{\gamma}{\# C} \sum_{g_{j} \in C} \operatorname{diver}\left(h, g_{j}\right)-\varepsilon_{t}(h)
$$

Until: the size of $C$ is $m$

Output: The selected classifier subset $C$

Fig. 1. Heuristic classifier subset selection

there is no straightforward criterion to measure the former. Presently, various pairwise diversity measures have been proposed, such as Disagreement measure, Q-statistics, Correlation coefficient and Kappa degree of agreement ([7]). We can choose any one of them and different diversity functions. On the whole, different diversity functions lead to similar performance in the experiments, although they may generate slightly different performance on different data sets. However, randomly selected classifier subset may counteract the training performance.

$$
\begin{aligned}
& \boldsymbol{\alpha}_{t}=\underset{\boldsymbol{\alpha}=\left(\alpha_{1}, \alpha_{2}, \ldots, \alpha_{m}\right) \in \mathbb{R}^{m}}{\arg \min } C\left(F_{t-1}+\sum_{k=1}^{k=m} \alpha_{k} h_{t_{k}}\right) \\
& d_{t+1}(i)=d_{t}(i) \exp \left(-y_{i} \sum_{k=1}^{k=m} \alpha_{t, k} h_{t_{k}}\left(\mathbf{x}_{i}\right)\right) / \sum_{j=1}^{j=N} d_{t}(j) \exp \left(-y_{j} \sum_{k=1}^{k=m} \alpha_{t, k} h_{t_{k}}\left(\mathbf{x}_{j}\right)\right) \\
& F_{t}(\mathbf{x})=F_{t-1}(\mathbf{x})+\sum_{k=1}^{k=m} \alpha_{t, k} h_{t_{k}}(\mathbf{x}) \\
& g_{t, k}=\sum_{\tau=1}^{\tau=t} \omega_{t, k, \tau} h_{\tau} \\
& \boldsymbol{\alpha}_{t}=\underset{\boldsymbol{\alpha}\left(=\alpha_{1}, \alpha_{2}, \ldots, \alpha_{m}\right) \in \mathbb{R}^{m}}{\arg \min } C\left(F_{t-1}+\sum_{k=1}^{k=m} \alpha_{k} g_{t, k}\right) \\
& d_{t+1}(i)=d_{t}(i) \exp \left(-y_{i} \sum_{k=1}^{k=m} \alpha_{t, k} g_{t, k}\left(\mathbf{x}_{i}\right)\right) / \sum_{j=1}^{j=N} d_{t}(j) \exp \left(-y_{j} \sum_{k=1}^{k=m} \alpha_{t, k} g_{t, k}\left(\mathbf{x}_{j}\right)\right) \\
& F_{t}(\mathbf{x})=F_{t-1}(\mathbf{x})+\sum_{k=1}^{k=m} \alpha_{t, k} g_{t, k}(\mathbf{x})
\end{aligned}
$$




\subsection{A New Criterion for PC-AB}

In this section, we discuss a more accurate evaluation for effective choice of classifiers. The basic idea is to approximate the cost function using a simple function and try to minimize the new function instead. For the classifiers $h_{t_{1}}, h_{t_{2}}, \ldots, h_{t_{m}}$, we define a $N \times m$ matrix $\mathbf{H}=\left\{y_{p} h_{t_{q}}\left(\mathbf{x}_{p}\right)\right\}_{1 \leq p \leq N, 1 \leq q \leq m}$. Its combination coefficients are represented by a vector $\mathbf{c}$. By Taylor expansion, we approximate the cost function as Eq.(14). The minimum of $K_{t}(\mathbf{H}, \mathbf{c})$ (denoted by $K_{t}(\mathbf{H})$, Eq. (16)) is achieved only when $\mathbf{c}=-\left(\mathbf{H}^{T} \nabla^{2} C(F) \mathbf{H}\right)^{-1} \mathbf{H}^{T} \nabla C(F)$.

$$
\begin{gathered}
C\left(F_{t}+\mathbf{H} \mathbf{c}\right) \approx C\left(F_{t}\right)+K_{t}(\mathbf{H}, \mathbf{c}) \\
K_{t}(\mathbf{H}, \mathbf{c})=\mathbf{c}^{T} \mathbf{H}^{T} \nabla C\left(F_{t}\right)+\frac{1}{2} \mathbf{c}^{T} \mathbf{H}^{T} \nabla^{2} C\left(F_{t}\right) \mathbf{H} \mathbf{c} \\
K_{t}(\mathbf{H}) \triangleq \min _{\mathbf{c} \in \mathbb{R}^{m}} K(\mathbf{H}, \mathbf{c})=-\frac{1}{2} \nabla C\left(F_{t}\right)^{T} \mathbf{H}\left(\mathbf{H}^{T} \nabla^{2} C\left(F_{t}\right) \mathbf{H}\right)^{-1} \mathbf{H}^{T} \nabla C\left(F_{t}\right)
\end{gathered}
$$

$\nabla^{2} C\left(F_{t}\right)$ (Eq. (17)) is the Hessian matrix of $C\left(F_{t}\right)$ and it is both symmetric and semi-definite. But $\mathbf{H}^{T} \nabla^{2} C\left(F_{t}\right) \mathbf{H}$ is always non-invertible. We slightly modify our criterion to $Q_{t}(\mathbf{H})$ by introducing a regularization item and discarding the constant factor. Eq.(18) corresponds to a modified goal function $\tilde{K}_{t}(\mathbf{H}, \mathbf{c})$ shown in Eq.(19). On the other hand, regularization is also helpful to control $\mathbf{c}$ in order to assure the validity of Taylor expansion. Next, we focus on how to minimize $Q_{t}(\mathbf{H})$ in order to achieve very promising descending potentialities.

$$
\begin{gathered}
\nabla^{2} C\left(F_{t}\right)_{p, q}= \begin{cases}d_{t}(p)\left(1-d_{t}(p)\right) & p=q \\
-y_{p} y_{q} d_{t}(p) d_{t}(q) & p \neq q\end{cases} \\
Q_{t}(\mathbf{H}) \triangleq \nabla C\left(F_{t}\right)^{T} \mathbf{H}\left(\mathbf{H}^{T} \nabla^{2} C\left(F_{t}\right) \mathbf{H}+\lambda \mathbf{I}\right)^{-1} \mathbf{H}^{T} \nabla C\left(F_{t}\right) \\
\tilde{K}_{t}(\mathbf{H}, \mathbf{c})=\mathbf{c}^{T} \mathbf{H}^{T} \nabla C\left(F_{t}\right)+\frac{1}{2} \mathbf{c}^{T} \mathbf{H}^{T} \nabla^{2} C\left(F_{t}\right) \mathbf{H} \mathbf{c}+\frac{\lambda}{2}\|\mathbf{c}\|_{2}^{2}
\end{gathered}
$$

First we can also replace the criterion Eq.(13) by our new criterion and use the same heuristic classifier subset selection strategy. Besides, the new criterion provides us a way to implement PC-AB2. Now we put all the member classifiers into $\mathbf{H}$. Since $\mathbf{H}^{T} \nabla^{2} C\left(F_{t}\right) \mathbf{H}+\lambda \mathbf{I}$ is symmetric and positive-definitive, we have orthogonal matrix $\boldsymbol{\Sigma}$ that satisfies $\boldsymbol{\Sigma}^{T}\left(\mathbf{H}^{T} \nabla^{2} C\left(F_{t}\right) \mathbf{H}+\lambda \mathbf{I}\right) \boldsymbol{\Sigma}=\boldsymbol{\Lambda}$, where $\boldsymbol{\Lambda}$ is a diagonal matrix of the eigenvalues of $\mathbf{H}^{T} \nabla^{2} C\left(F_{t}\right) \mathbf{H}+\lambda \mathbf{I}$. Therefore $Q_{t}(\mathbf{H})$ can be simplified as Eq.(20), where $\boldsymbol{\Lambda}=\operatorname{diag}\left\{\lambda_{1}, \lambda_{2}, \ldots, \lambda_{m}\right\}$ and $\boldsymbol{\Sigma}^{T} \mathbf{H}^{T} \nabla C\left(F_{t}\right)$ is a $m$-dimensional vector represented by $\left[l_{1}, l_{2}, \ldots, l_{m}\right]^{T}$. The detailed algorithm to construct classifier combinations at each iteration is shown in Fig 2, then follow Eq.(12) (a)-(d) to adjust sample distribution and member classifiers' coefficients.

$$
Q_{t}(\mathbf{H})=\nabla C\left(F_{t}\right)^{T} \mathbf{H} \boldsymbol{\Sigma} \boldsymbol{\Lambda}^{-1} \boldsymbol{\Sigma}^{T} \mathbf{H}^{T} \nabla C\left(F_{t}\right)=\sum_{k=1}^{k=m} \frac{l_{k}^{2}}{\lambda_{k}}
$$


Input: $t$ available classifiers $h_{1}, h_{2}, \ldots, h_{t}$ and a positive integer $m$, where $1 \leq m \leq t$. Step1: Calculate $\nabla^{2} C\left(F_{t}\right)$ as Eq. (17).

Step2: Find a orthogonal matrix $\boldsymbol{\Sigma}$ and a diagonal matrix $\boldsymbol{\Lambda}=\operatorname{diag}\left\{\lambda_{1}, \lambda_{2}, \ldots, \lambda_{m}\right\}$ that satisfies $\boldsymbol{\Sigma}^{T}\left(\mathbf{H}^{T} \nabla^{2} C\left(F_{t}\right) \mathbf{H}+\lambda \mathbf{I}\right) \boldsymbol{\Sigma}=\boldsymbol{\Lambda}$ and let $\left[l_{1}, l_{2}, \ldots, l_{t}\right]^{T}=\boldsymbol{\Sigma}^{T} \mathbf{H}^{T} \nabla C\left(F_{t}\right)$. Step3: Sort $\left\{\frac{l_{k}^{2}}{\lambda_{k}}\right\}_{1 \leq k \leq t}$ in descending order as $\frac{l_{j_{1}}^{2}}{\lambda_{j_{1}}} \geq \frac{l_{j_{2}}^{2}}{\lambda_{j_{2}}} \geq \cdots \geq \frac{l_{j_{t}}^{2}}{\lambda_{j_{t}}}$.

Output: $g_{t, k}=\sum_{\tau=1}^{\tau=t} \sigma_{\tau, j_{k}} h_{\tau}, 1 \leq k \leq m$, where $\boldsymbol{\Sigma}=\left\{\sigma_{p, q}\right\}_{1 \leq p \leq t, 1 \leq q \leq t}$.

Fig. 2. Generating classifier linear combinations for PC-AB2

\section{Experiments}

\subsection{Experiment Configurations}

We performed the experiments on 12 UCI machine learning data sets ('balancescale', 'breast-cancer-w', 'credit-a', 'credit-g', 'diabetes', 'heart-c', 'heart-statlog', 'ionosphere', 'kr-vs-kp', 'sonar', 'splice', 'waveform5000'). The base learner is decision stump implemented by WEKA. We use a 10-fold cross validation to compare the training and testing performance of standard AdaBoost and three proposed implementation strategies: 1)PCAB1D: PC-AB1 with heuristic classifier selection based on the criterion described in Eq. (13) $(\gamma=1, m=5)$; 2)PCAB1Q: PC-AB1 with heuristic classifier selection based on the criterion described in Eq.(18) $(m=5)$; 3)PCAB2Q: PC-AB2 with given classifier combinations as described in section $3.3(\lambda=0.001, m=5) . m$ controls algorithm's complexity, so larger $m$ will lead to faster training error convergence but more time consumption. $\gamma$ and $\lambda$ are both set by experience without optimization step.

\subsection{Experiment Results}

The results are shown in Fig 3(a)-(p) (Due to the page limitation, only part of the results are shown). In each graphic, we compare the ensemble error variations of AdaBoost (blue), PCAB1D (red), PCAB1Q (green) and PCAB2Q (black).

From the experiments, we find that PCAB1D, PCAB1Q and PCAB2Q all outperform AdaBoost and PCAB2Q always has the overall best performance. The diversity based classifier selection, as we have discussed, uses an empirical evaluation to select a classifier subset, so PCAB1D performs worse than PCAB1Q, which adopts a more accurate criterion to measure the cost function descent potentialities. Although PCAB1Q and PCAB2Q both select descent directions by the same criterion $Q_{t}, \mathrm{PCAB} 2 \mathrm{Q}$ develops a more aggressive way by using the linear combinations of all the previous classifiers, it is believed that PCAB2Q can achieve better performance than the other two strategies, which is also substantiated by the experiments. Generalization performance is also improved in $\mathrm{PC}-\mathrm{AB}$, it can substantiate that the cost function not only bounds the training error, but also potentially controls the generalization risk. 


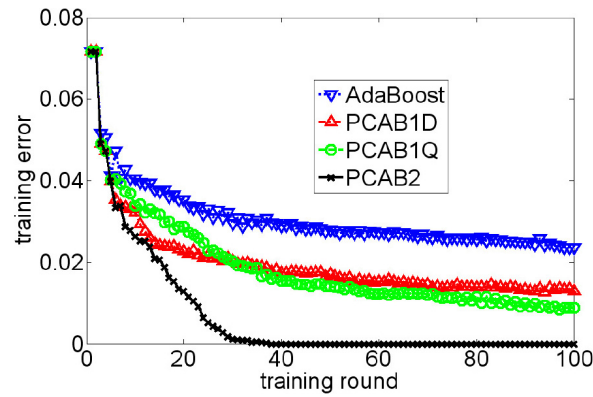

(a) training of breast-cancer-w

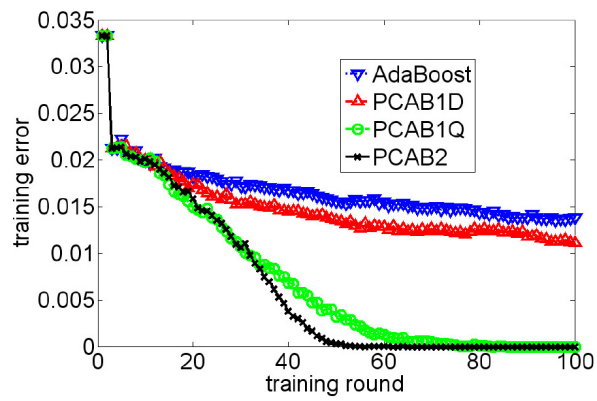

(c) training of heart-c

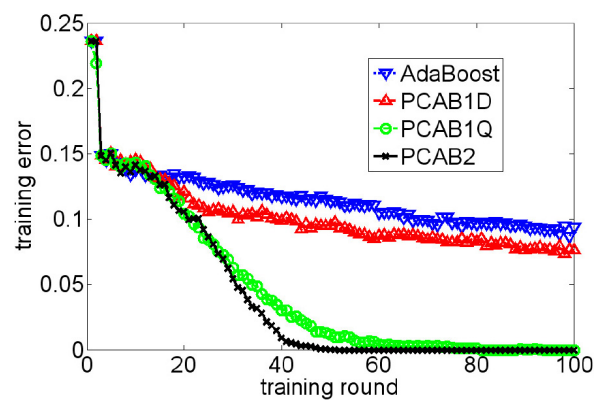

(e) training of heart-statlog

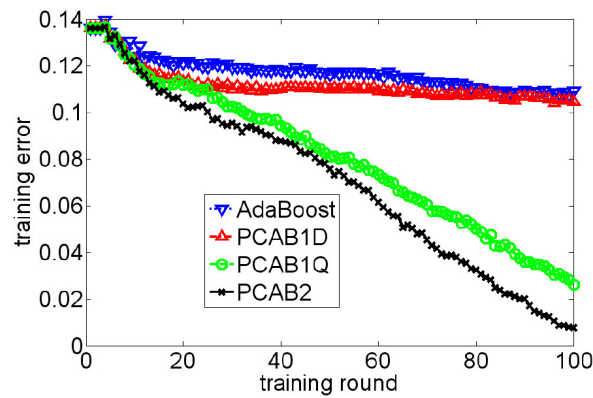

(g) training of credit-a

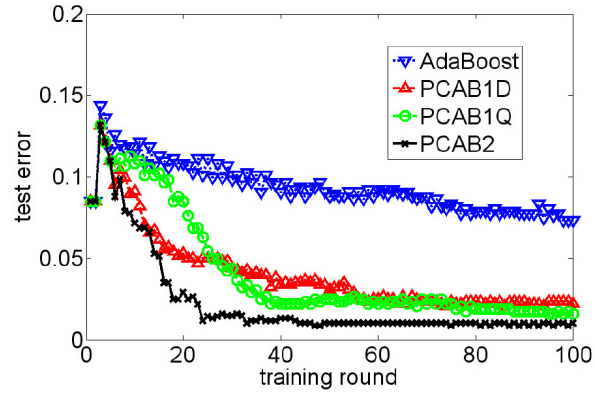

(b) testing of breast-cancer-w

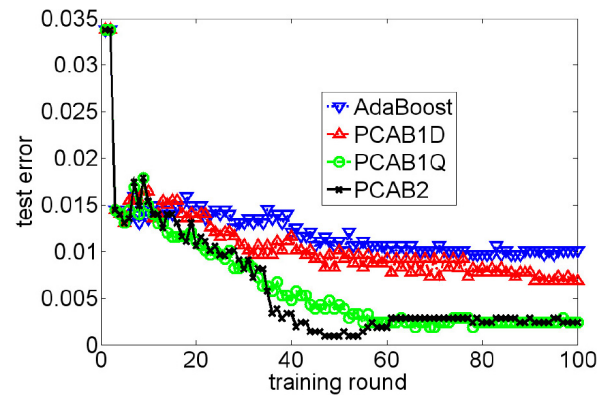

(d) testing of heart-c

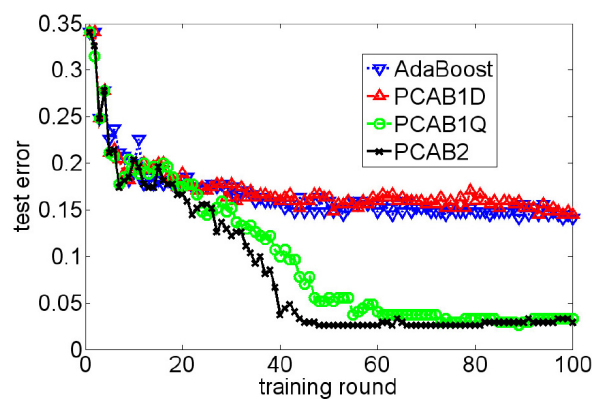

(f) testing of heart-statlog

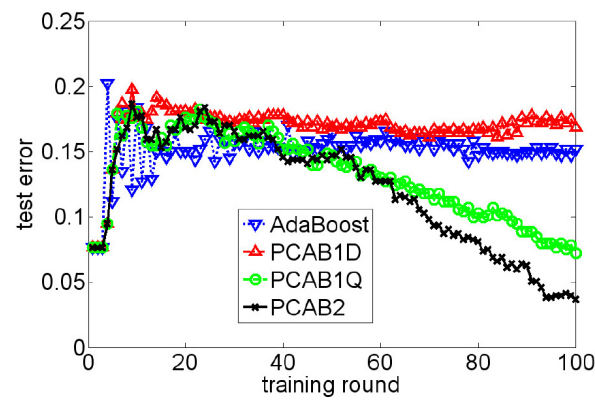

(h) testing of credit-a

Fig. 3. Experiment results on selected UCI data sets 


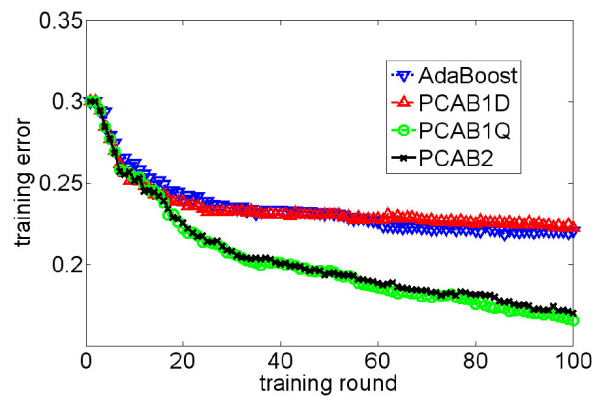

(a) training of credit-g

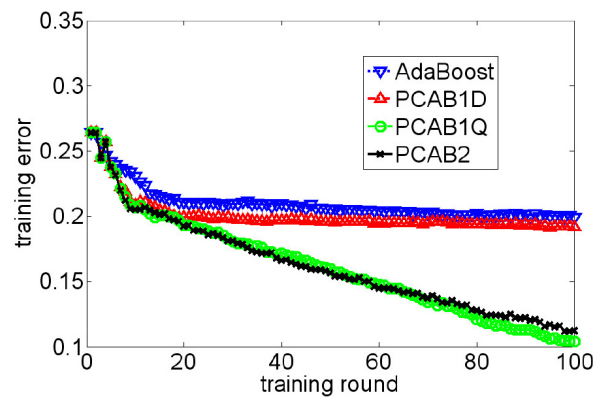

(c) training of diabetes

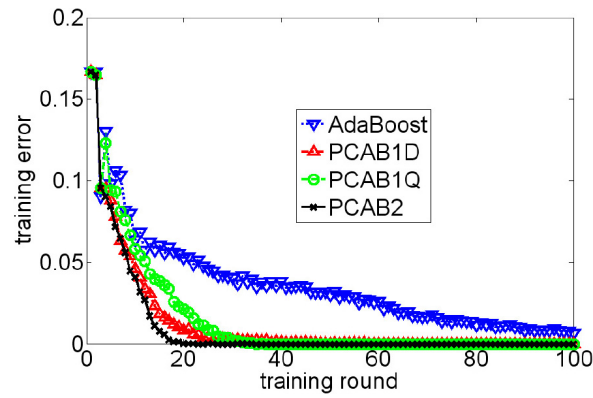

(e) training of ionosphere

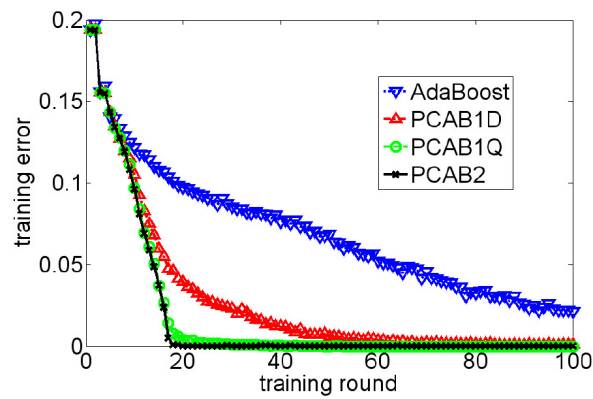

(g) training of balance-scale

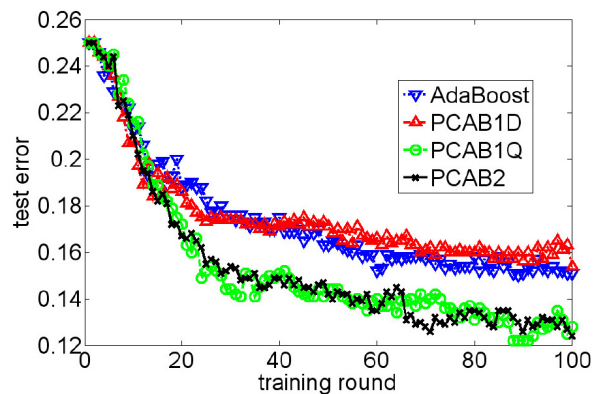

(b) testing of credit-g

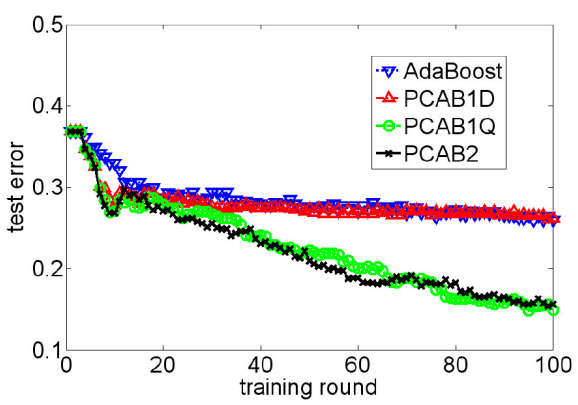

(d) testing of diabetes

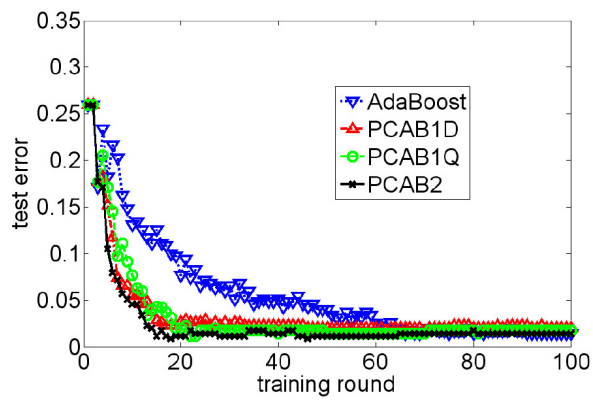

(f) testing of ionosphere

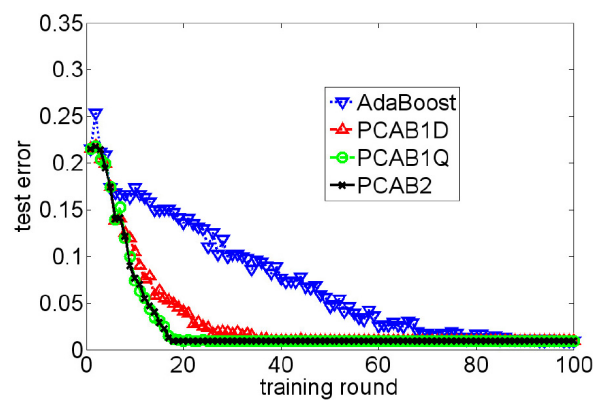

(h) testing of balance-scale

Fig. 3. (continued) 


\section{Conclusion}

We propose a practical way to improve the convergence of AdaBoost by seeking for a distribution update method that reduces the correlations between the previous classifiers and the new distribution. Comparing with 'totally corrective', our method pursues a 'partially corrective' distribution to balance the requirement of considering more previous classifiers' information and time complexity.

We propose two types of PC-AB: PC-AB1 aims to select a subset from the previous classifiers and make the new distribution uncorrelated to these classifiers; in PC-AB2, the new distribution is required to be corrective to a set of classifier linear combinations. Lagrange duality theory shows that the proposed methods actually focus on how to construct a more effective descent direction by adequately considering the previously adopted directions. Thus the previous member classifiers' coefficients can be iteratively modified to achieve better ensemble performance. Experiments show that our method can significantly improve both training and generalization performance of standard AdaBoost.

\section{References}

1. Freund, Y., Schapire, R.E.: A Decision-Theoretic Generalization of On-line Learning and an Application to Boosting. In: Vitányi, P.M.B. (ed.) EuroCOLT 1995. LNCS, vol. 904, pp. 23-37. Springer, Heidelberg (1995)

2. Mason, L., Baxter, J., Bartlett, P.L., et al.: Boosting Algorithms as Gradient Descent. In: Advances in Neural Information Processing Systems (NIPS 1999), pp. 512-518 (1999)

3. Friedman, J.H., Hastie, T., Tibshirani, R.: Additive Logistic Regression: A Statistical View of Boosting. Annals of Statistics 28(2), 337-407 (1998)

4. Li, L., Abu-Mostafa, Y.S., Pratap, A.: CGBoost: Conjugate Gradient in Function Space. Technical Report CaltechCSTR: 2003.007, Learning Systems Group, California Institute of Technology (August 2003)

5. Kivinen, J., Warmuth, M.K.: Boosting as Entropy Projection. In: 12th Annual Conference on Computational Learning Theory (COLT 1999), pp. 134-144 (1999)

6. Bühlmann, P., Yu, B.: Boosting with the $L_{2}$-Loss: Regression and Classification. Journal of American Statistical Association 98, 324-339 (2003)

7. Kuncheva, L.I., Whitaker, C.J.: Measures of Diversity in Classifier Ensembles and Their Relationship with the Ensemble Accuracy. Machine Learning 51(2), 181-207 (2003) 